EPJ Web of Conferences 69, 00016 (2014)

DOI: $10.1051 /$ epjconf/20146900016

(C) Owned by the authors, published by EDP Sciences, 2014

\title{
SPY: a new scission-point model based on microscopic inputs to predict fission fragment properties
}

\author{
Stefano Panebianco ${ }^{1}$, Nöel Dubray ${ }^{2}$, Stéphane Goriely ${ }^{3}$, Stéphane Hilaire ${ }^{2}$, Jean-François Lemaître ${ }^{1}$ \\ and Jean-Luc Sida ${ }^{1}$ \\ ${ }^{1}$ CEA Centre de Saclay, Irfu, 91191 Gif-sur-Yvette, France \\ ${ }^{2}$ CEA, DAM, DIF, 91297 Arpajon, France \\ ${ }^{3}$ Institut d'Astronomie et d'Astrophysique, CP-226, Université Libre de Bruxelles, 1050 Brussels, \\ Belgium
}

\begin{abstract}
Despite the difficulty in describing the whole fission dynamics, the main fragment characteristics can be determined in a static approach based on a so-called scission-point model. Within this framework, a new Scission-Point model for the calculations of fission fragment Yields (SPY) has been developed. This model, initially based on the approach developed by Wilkins in the late seventies, consists in performing a static energy balance at scission, where the two fragments are supposed to be completely separated so that their macroscopic properties (mass and charge) can be considered as fixed. Given the knowledge of the system state density, averaged quantities such as mass and charge yields, mean kinetic and excitation energy can then be extracted in the framework of a microcanonical statistical description. The main advantage of the SPY model is the introduction of one of the most up-to-date microscopic descriptions of the nucleus for the individual energy of each fragment and, in the future, for their state density. These quantities are obtained in the framework of HFB calculations using the Gogny nucleon-nucleon interaction, ensuring an overall coherence of the model. Starting from a description of the SPY model and its main features, a comparison between the SPY predictions and experimental data will be discussed for some specific cases, from light nuclei around mercury to major actinides. Moreover, extensive predictions over the whole chart of nuclides will be discussed, with particular attention to their implication in stellar nucleosynthesis. Finally, future developments, mainly concerning the introduction of microscopic state densities, will be briefly discussed.
\end{abstract}

\section{Introduction}

It is often said that nuclear fission is a well-known process. There is no doubt that, since its discovery by Hahn and Strassmann in 1938 [1], a huge scientific effort, both theoretical and experimental, has been pursued by several generations of physicists, leading to a rather good understanding of the most general properties of this process. In addition, the main observables relevant for nuclear energy applications have been extensively measured and provide a reliable foundation for the engineering design and operation of present nuclear plants. On the other hand, almost 75 years of research in 
fission physics have raised a large number of unsolved questions about the "fine structure" of the mechanism, leading to a generally admitted statement: up to now, there is no global and comprehensive physics theory which is able to correctly describe and, more important, to reliably predict the whole set of observables of a compound nucleus fission process. Having in mind the fact that nuclear fission is probably the only process deeply involving all the different aspects of nuclear physics (from the basic nuclear structure to the N-body collective interactions), it is evident that it constitutes an ideal "nuclear physics laboratory" whose results have a direct impact on a wide number of nuclear physics fields.

We dispose of a large variety of models and theories which address these questions, all trying to solve the nuclear time-dependent many-body problem for the compound excited nucleus, starting from saddle point conditions down to, at least, the configuration where the separation of the fission fragments has occurred, the so-called scission point. There are globally two classes of methods to model the fission of a nucleus [2]. The first one is the phenomenological approach which is based on the physical interpretation of experimental data with quite simple theoretical tools which allows short computation time. However, this approach makes use of many parameters fitted on experimental data which limits the predictive power when going far from the region where data is available. The second one is the microscopic approach. It is based on a full quantum treatment from a given microscopic nucleon-nucleon interaction to describe the system, both from the static and dynamic point of view. Therefore, even though the coherence of this approach is ensured over the whole nuclear chart, the needed computation time is clearly a major limitation.

Therefore, we chose an intermediate approach making use of one of the most up-to-date microscopic descriptions of nuclear structure, implemented as an input in the framework of a rather simplified approach, a scission-point model. This model, called SPY (Scission Point Yields) allows determining of the fission fragment properties (mass and charge yields, kinetic and excitation energies) from a statistical treatment of all static configurations which are energetically reachable at scission.

\section{The SPY model}

SPY is a renewed version of the well-known scission-point model from Wilkins [3] based on the basic assumption that fission fragment distributions can be determined from the available energy of the different configurations at or near the scission point, taking into account a proper statistical description. Given this static picture of the scission configuration, the compound nucleus formation mechanism and the system dynamics from saddle to scission are not taken into account. In this approach, all fragments properties (mass, charge and deformation) are therefore considered fixed at scission. The system is modeled by two coaxial ellipsoids of revolution with sharp frontiers and separated by a fixed distance $d$ (Figure 1). Each nucleus is characterized by its neutron $(\mathrm{N})$ and proton number $(Z)$ and its deformation parameter $(\beta)$. Thermodynamical equilibrium is supposed to be reached at scission, implying a statistical equilibrium among the system degrees of freedom. In addition the two fragments are considered to be isolated and all the states that are energetically reachable are equiprobable. Within these hypotheses, the scission configuration can be treated as a microcanonical statistical ensemble in order to calculate average observables such as fission yields, mean kinetic and excitation energies of the fragments. 


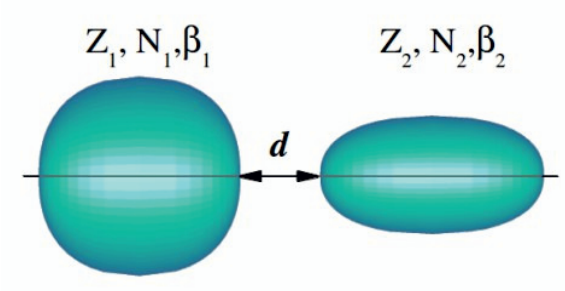

Figure 1.Schematic description of the two-fragment system at the scission point.

\subsection{The energy balance}

Once the scission configuration is defined, the first stage of the calculation for a given fissioning nucleus is to perform a proper energy balance for all possible fragmentations (around 1000) as a function of the deformation parameter of the two fragments. As the competition between symmetric and asymmetric fission is related to shell effects in the fragments, it is essential to describe them well up to very deformed shapes. The available energy $\left(E_{\text {avail }}\right)$ calculation is based, as it is the case in Wilkins model, on an energy balance between the compound nucleus energy $\left(E_{C N}\right)$ and the intrinsic excitation energy of the system of the two nascent fragments (Equation 1). This latter contribution is obtained as the sum of the individual energies of the two fragments and their interaction energy. The individual energy $\left(E_{H F B}\right)$ is extracted from one of the most up-to-date descriptions of microscopic nuclear structure. In particular, we use the Amedee database [4,5] which contains the potential energy as a function of the deformation over a large range of deformations, from very oblate to very prolate shapes, and for all the nuclei over the whole nuclear chart. This potential energy is calculated in the framework of the Hartree-Fock-Bogoliubov (HFB) approach using the Gogny D1S nucleon-nucleon interaction [6]. This is one of the major improvements brought by SPY, compared to the existing scission-point models. It has the advantage of giving access to the absolute value of the available energy and gets rid of all temperature parameters of the Wilkins-like models. Thanks to this microscopic description, the model gives access to the fission properties over the whole chart of nuclides. In order to keep the computing time reasonable, this microscopic data is integrated into the SPY code structure on the form of a large database. Finally, the interaction energy between the two fragments is composed of two terms: a Coulomb interaction term $\left(E_{\text {Coul }}\right)$ [7] and a proximity nuclear interaction $\left(E_{\text {nucl }}\right)$ [8]. The interaction energy is the only term in the balance that depends on the scission distance which is kept fixed at $5 \mathrm{fm}$ in all our calculations. A fragmentation is energetically achievable if the available energy is negative.

$$
\begin{aligned}
& E_{\text {avail }}=E_{H F B 1}\left(Z_{1}, N_{1}, \beta_{1}\right)+E_{H F B 2}\left(Z_{2}, N_{2}, \beta_{2}\right) \\
& +E_{C o u l}\left(Z_{1}, N_{1}, \beta_{1}, Z_{2}, N_{2}, \beta_{2}, d\right)+E_{\text {nucl }}\left(Z_{1}, N_{1}, \beta_{1}, Z_{2}, N_{2}, \beta_{2}, d\right) \\
& -E_{C N}
\end{aligned}
$$

As an example, the available energy as a function of the two fragment deformations, calculated for two scission configurations in the thermal neutron-induced fission of ${ }^{235} \mathrm{U}$, is presented in Figure 2 . The most probable asymmetric fragmentation (Figure 2 left) reaches its energy minimum at around $-40 \mathrm{MeV}$ and is characterized by one spherical fragment (the double magic ${ }^{132} \mathrm{Sn}$ ) and a rather deformed fragment $\left({ }^{104} \mathrm{Mo}\right)$. In contrast, the symmetric splitting is characterized by a higher available energy (around $-35 \mathrm{MeV}$ ) and two deformed fragments. The asymmetric configuration is hence energetically favored with respect to the symmetric one, as expected in ${ }^{235} \mathrm{U}$ thermal fission. 

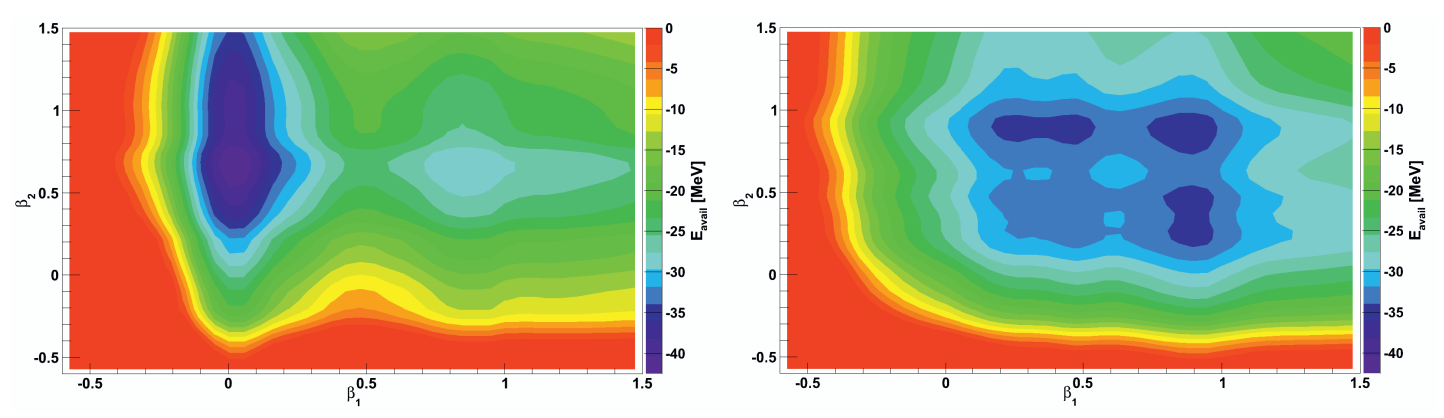

Figure 2. Available energy as a function of the fragment deformations $\beta_{1}$ and $\beta_{2}$ for asymmetric $\left({ }^{132} \mathrm{Sn}+{ }^{104} \mathrm{Mo}\right)$ on the left and symmetric fragmentation $\left({ }^{118} \mathrm{Pd}+{ }^{118} \mathrm{Pd}\right)$ in ${ }^{235} \mathrm{U}$ thermal fission.

\subsection{The statistical treatment}

In a microcanonical statistical description, all energetically reachable states are equiprobable. Therefore, all system averaged observables can be calculated from the intrinsic excitation of the twofragment system by a simple counting of all available states for all possible fragmentations. Actually, the knowledge of the exact energy of all states is not necessary and only their number (or density) for a given configuration is needed. In our approach, a supplementary hypothesis is made: the number of states $\pi$ reachable by the system for a given configuration is defined as the product of the state densities of the two fragments $\left(\rho_{l}\right.$ and $\left.\rho_{2}\right)$ where a fraction $x$ of available energy goes to fragment 1 and a fraction $(1-x)$ goes to fragment 2 :

$$
\pi\left(Z_{1}, N_{1}, Z_{2}, N_{2}, \beta_{1}, \beta_{2}, x\right)=\rho_{1}\left(x\left|E_{\text {avail }}\right|\right) \rho_{2}\left((1-x)\left|E_{\text {avail }}\right|\right) \delta E^{2}
$$

The probability of a given configuration is then given by the number of states accessible to this configuration, divided by the total number of states of the system; therefore it is proportional to $\pi$. Finally, the total probability $P$ to obtain a given fragmentation is obtained by integrating over the two deformation parameters and all energy partitions:

$$
P\left(Z_{1}, N_{1}, Z_{2}, N_{2}\right)=\int_{-0.6}^{1.5} \int_{-0.6}^{1.5} \int_{0}^{1} \pi\left(Z_{1}, N_{1}, Z_{2}, N_{2}, \beta_{1}, \beta_{2}, x\right) d \beta_{1} d \beta_{2} d x
$$

This probability, normalized to $200 \%$, gives the production yield of each fission fragment and gives access to the averaged observables like the mean kinetic and excitation energy.

Presently, the state density of the fission fragments used in the SPY model is of a Fermi gas type from a generalized superfluid model [9]. This state density is independent of the fragment deformation and is a simple exponential function of the intrinsic energy $E$ :

$$
\rho(E) \propto e^{2 \sqrt{a E}}
$$

where $a \approx A / 8$ is the level density parameter and $A$ is the mass of the nucleus. Since the statistical treatment with a Fermi gas type state density does not introduce any structure in the available energy distribution, to first order the most probable fragmentation is characterized by the highest energy available to the system. In the conclusions, ongoing developments to improve the state density description will be briefly discussed. 


\section{$\mathrm{CNR} * 13$}

\section{Results}

The SPY model was first applied to two "standard" cases that unavoidable when dealing with nuclear fission [10]. In Figure 3, the fission fragment mass yields from the reactions ${ }^{235} \mathrm{U}\left(\mathrm{n}_{\mathrm{th}}, \mathrm{f}\right)$ and ${ }^{252} \mathrm{Cf}(\mathrm{sf})$ are presented and compared to evaluated data (from the JEFF-3.1.1 and ENDF/B-VII.1 data libraries) and to the GEF model [11], essentially based on phenomenology.

Given the strong hypothesis and the absence of tuneable parameters, the SPY calculation can be considered as satisfactory. The asymmetric character of the fission mass distribution is well reproduced. In the case of ${ }^{235} \mathrm{U}$ fission, the presence of a rather sharp peak at $A=132$ is the manifestation of the too strong influence of the spherical shell effects of ${ }^{132} \mathrm{Sn}$. This effect is less pronounced in the fragment mass distribution from ${ }^{252} \mathrm{Cf}$ spontaneous fission because of the presence of higher mass fragments exhibiting rather strong shell effects in competition with the doubly magic ${ }^{132} \mathrm{Sn}$ nucleus. Finally, the lack of fission dynamics is visible from the too small width of the distributions.

If the lack of dynamics is clearly a limitation of this approach, the precise calculation of the available and interaction energy of each fragmentation leads to quite powerful predictions on the fission fragment kinetic (Figure 4) and excitation energy. The comparison to experimental data and the GEF model for ${ }^{235} \mathrm{U}\left(\mathrm{n}_{\mathrm{th}}, \mathrm{f}\right)$ shows that the general trend of the fission fragment kinetic energies as a function of their mass is well reproduced, in particular the very characteristic plateau around mass number 125. A $5 \mathrm{MeV}$ shift compared to the data is expected since, for the time being, no pre-scission energy is calculated and therefore the absolute value of the kinetic energy depends on the choice of the scission distance.

$$
{ }^{235} \mathrm{U}\left(\mathrm{n}_{\mathrm{th}}, \mathrm{f}\right)
$$

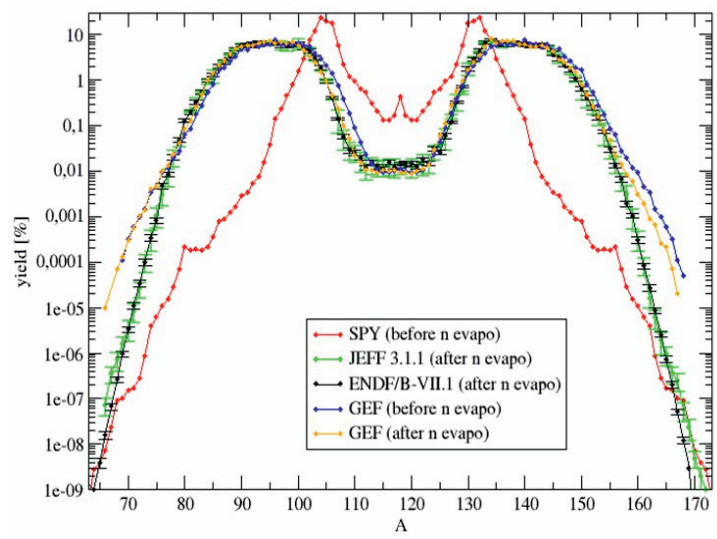

${ }^{252} \mathrm{Cf}(\mathrm{sf})$

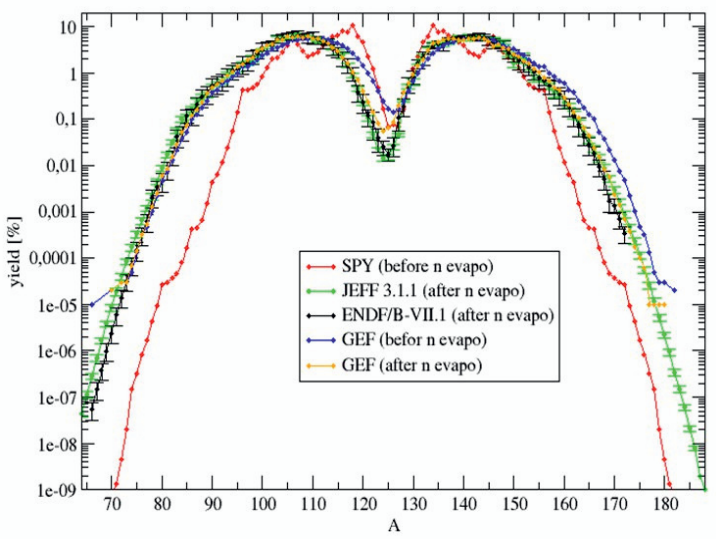

Figure 3. Fission fragment mass yields from SPY in ${ }^{235} \mathrm{U}$ thermal fission (left plot) and ${ }^{252} \mathrm{Cf}$ spontaneous fission (right). The SPY model (which does not treat neutron evaporation) is compared to the GEF model [11] (calculated before and after neutron evaporation) and the JEFF-3.1.1 and ENDF/B-VII.1 nuclear data libraries (which are evaluated from experimental yields measured after neutron evaporation).

The most important improvement brought by the microscopic input in this scission-point model is a powerful predictive power for fissioning nuclei far from the experimentally known actinide region where all the models based on phenomenology are tuned. Moreover, the fast treatment of thousands of calculated HFB energy potentials makes the necessary computing time extremely low. Indeed, SPY is presently the fastest microscopically-based fission model available on the market and has been already applied to two rather exotic physics cases where fission is involved. 


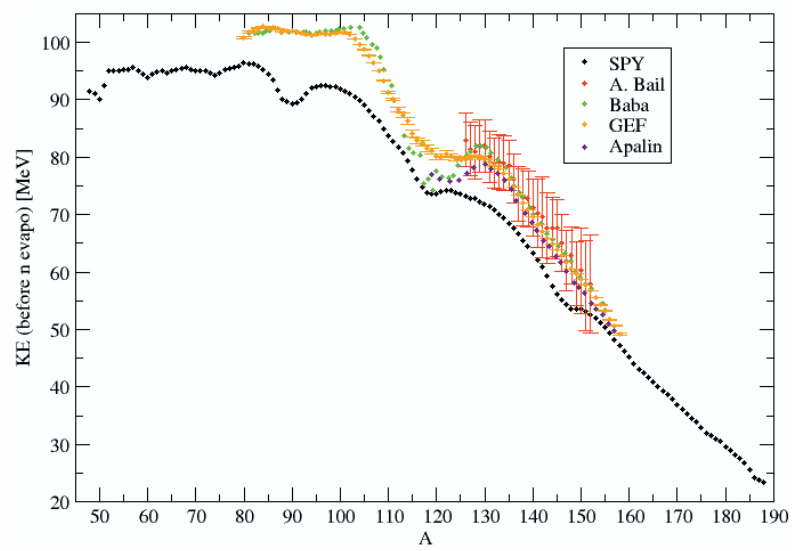

Figure 4. Kinetic energy of fission fragments as a function of their mass in ${ }^{235} \mathrm{U}$ thermal fission. The SPY prediction is compared to the GEF model [11] (calculated before evaporation) and a set of experimental data [1214].

\subsection{The asymmetric fission of ${ }^{180} \mathrm{Hg}$}

The position of the heavy mass peak in the thermal neutron-induced fission of actinides is known to be rather independent of the parent nucleus. This has very often been interpreted as a strong influence of the structure of the nascent fragments, for instance the heavy one for these systems. According to this observation, the absence of strong shell structures of the fragments for fission nuclei in the $\mathrm{Pb}$ region leads to a natural symmetric splitting. Quite recently, a surprising result appeared in the fission of ${ }^{180} \mathrm{Hg}$ where an asymmetric fragment mass distribution was found [15]. In a crude approximation, the symmetric fragmentation was expected to be favored since it would lead to the formation of two nuclei stabilized by a semi-magic $(Z=40)$ and a magic shell closure $(N=50)$. Soon after the publication of this unexpected result, new model calculations, involving the evolution of the energy potential between the parent nucleus ground state to the final state of separated nuclei [16], were able to describe the asymmetric fission of ${ }^{180} \mathrm{Hg}$ without considering explicitly the nascent fragment structure. This would have implied that the mass asymmetry is no longer driven by the structure of the nascent fragments and therefore that the scission-point model would not have been suitable to the mercury isotopes.

Despite this first conclusion, we were able to show that, as for fission of the actinides, the asymmetric split in the ${ }^{180} \mathrm{Hg}$ fission can be interpreted in the framework of a microscopically-based scission-point model such as SPY on the sole basis of the nuclear structure of the daughter nuclei [17]. Moreover, we could show that this approach helps to qualitatively understand the evolution from asymmetric to symmetric fission for intermediate mass nuclei as well as heavy actinides. This proves that the mass asymmetry in fission is essentially driven by the fragment's microscopic structure and in particular by its deformed shell effects.

\subsection{The role of fission in stellar nuclesynthesis}

The SPY approach has recently made possible the calculation of the fragment properties for thousands of fissioning nuclei (from proton to neutron drip lines) in a rather fast computer time (a few days with ten processors). This calculation gives access, for the first time, to an extensive study of the impact of nuclear structure on the fission yields of nuclei that are inaccessible for the majority of the models available on the market [10]. We could show that, like in the asymmetric fission of actinides, there is another region of asymmetric fission around $Z_{C N}=105$ and $N_{C N}$ between 170 and 180 where the double magic nucleus ${ }^{132} \mathrm{Sn}$ is the light fragment and the heavy fragment is even more deformed than the light fragment in the actinide region. Moreover, we found three regions of symmetric fission: the first two 
are related to very proton-rich and very neutron-rich actinides while the third one concerns extremely neutron-rich nuclei, close to the neutron drip-line. In all these regions the two fragments are slightly deformed.

This exotic region was found to present a very peculiar behavior and a very important impact on nuclear astrophysics. It is known that fission recycling plays a crucial role in stellar nucleosynthesis, in particular for the production of heavy r-process elements in neutron star merger ejecta. On the contrary, the production mechanism of the lightest $110<\mathrm{A}<170$ nuclei, essentially the rare-earth elements, has been rather unclear up to now and extremely dependent on fission properties, including in particular the fission fragment yields. The SPY model predictions (fission fragment yields and mean evaporated neutrons per fragmentation deduced from the excitation energy) have been integrated to one of the most advanced neutron star merger simulations of the r-process [19-21]. It was found that the SPY predictions for nuclei of mass around 165 (Figure 5) are the only ones able to explain the origin of the rare-earth peak by the r-process, in contrast to the more phenomenological fission models which all predict a symmetric fission distribution for the progenitor nuclei and therefore underestimate the production of fission fragments in this region. This very important result comes from the fact that fissioning nuclei of mass 278 are the main progenitors of the rare-earth nuclei in the decompression of neutron star matter. These nuclei present an unexpected doubly asymmetric fission mode with a characteristic four-hump pattern (Figure 6). Such fragment yields, which have never been observed experimentally, are due to shell effects at large deformations of the neutron-rich fragments favored by the fission of $A=278$ isobars. Finally, the two asymmetric fission modes have also been observed on the potential energy surface obtained from a full microscopic HFB calculation for the ${ }^{278} \mathrm{Cf}$ nucleus in a bidimensional deformation subspace [19]. The SPY prediction of this unexpected doubly asymmetric fission opens new perspectives in theoretical and experimental nuclear physics regarding specific fission modes related to the nuclear structure properties of exotic nuclei.

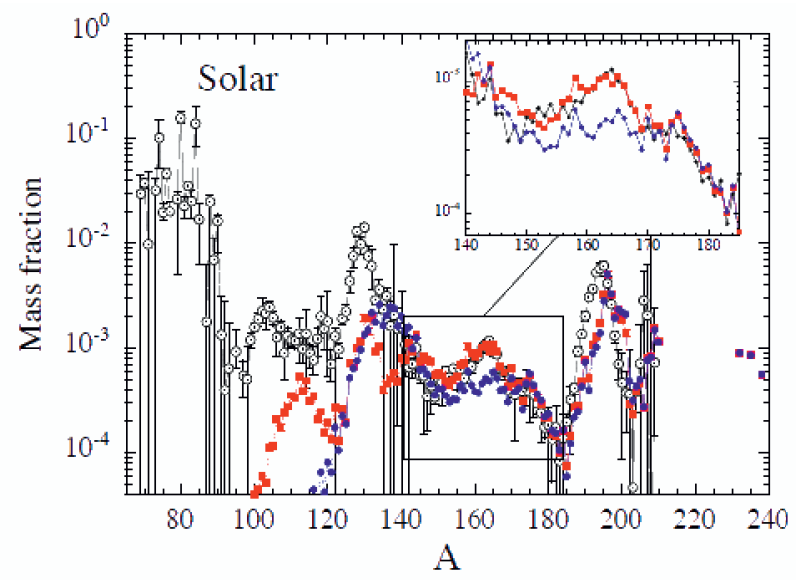

Figure 5. Final matter abundance distribution from neutron star ejecta as a function of the mass. The red squares are obtained using the fission distribution calculated with SPY. They are compared to the solar abundances [18] (dotted circles) and the same calculation performed using the fission distributions from the GEF model [11]. 


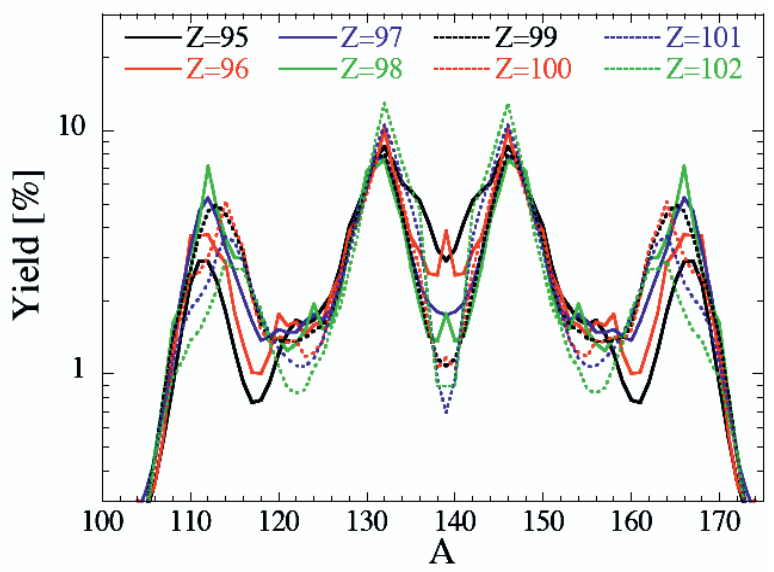

Figure 6. Fission fragment mass yields calculated wit SPY for eight isobars of mass 278.

\section{Conclusion and perspectives}

A new scission-point model, called SPY and based on a static and statistical description of the fission process, has been developed using of one of the most up-to-date microscopic descriptions of nuclear structure. SPY is able to explain the competition between asymmetric and symmetric fission modes from actinides to the light mercury isotopes in terms of the influence of deformed shell effects. Moreover, the richness of the microscopic input data opens the possibility to study the fission of unexplored regions of the nuclear chart. In particular, SPY predicts a new zone of doubly asymmetric fission for $\mathrm{A}=278$ isobars. The fission fragments produced by these nuclei are able to describe, for the first time, the production of rare-earth nuclei in the neutron star merger r-process.

Encouraged by these first results, a certain number of improvements are presently under development. First, an exact calculation of the Coulomb potential using a double-folding technique will provide a more solid description of the interaction energy and, eventually coupled to a prescission term, will provide a better determination of the kinetic energy. In addition, a large effort is being put into the integration of state densities obtained from the same microscopic calculations used for the available energy determination. These new densities, which are dependent on the nuclear deformation, might partly counterbalance the too strong shell effects around magicity and will provide an overall coherence of the model. Moreover, the integration of the finite temperature HFB calculation for the individual energy and the state density will give access to dissipation properties of the scissioning system and will provide an extension of the domain of validity of the model in terms of excitation energy (above $15 \mathrm{MeV}$ ).

\section{References}

1. O. Hahn and F. Strassman, Naturwissenschaften 27, 11 (1939)

2. C. Wagemans, The Nuclear Fission Process, Ed. C. Wagemans, CRC Press (1991)

3. B. D. Wilkins et al., Phys. Rev. C 14, 5 (1976)

4. S. Hilaire and M. Girod, Eur. Phys. J. A 33, 237 (2007)

5. http://www-phynu.cea.fr/HFB-Gogny.htm

6. J. Decharge and D. Gogny, Phys. Rev. C 21, 1568 (1980)

7. S. Cohen and W. Swiatecki, Ann. Phys. (NY) 19, 67 (1962)

8. J. Błocki, J. Randrup, W. J. Swiatecki, and C. F. Tsang, Ann. Phys. (NY) 105, 427 (1977)

9. A. V. Ignatyuk, J. L. Weil, S. Raman, and S. Kahane, Phys. Rev. C 47, 1504 (1993)

4. J. F. Lemaître et al., Proc. «Fission 2013», Caen (France), 28-31/05/2013

5. K.-H. Schmidt, B. Jurado, JEF/DOC 1423, OECD Nuclear-Energy Agency, Paris (2012) 


\section{$\mathrm{CNR} * 13$}

6. A. Bail, PhD thesis, Université Bordeaux I (2009)

7. H. Baba et al., Nucl. Scien. And Techn. 34, 871 (1997)

8. V. F. Apalin et al., Nucl. Phys. 71 3, 546 (1965)

9. A. Andreyev et al., Phys. Rev. Lett. 105, 252502 (2011)

10. P. Möller et al., Phys. Rev. C 85, 024306 (2012)

11. S. Panebianco et al., Phys. Rev. C 86, 064601 (2012)

12. S. Goriely, Astron. Astrophys. 342, 881 (1999)

13. S. Goriely et al., arXiv:1311.587 [astro-ph.SR], accept. for public. in Phys. Rev. Lett. (2013)

14. S. Goriely, A. Bauswein, and H.-T. Janka, Astrophys. J. Lett. 738, L32 (2011)

15. A. Bauswein, S. Goriely, and H.-T. Janka, Astrophys. J. 773, 78 (2013) 